\title{
Emotion Processing in the Visual Brain: A MEG Analysis
}

\author{
Peter Peyk · Harald T. Schupp · Thomas Elbert • \\ Markus Junghöfer
}

Accepted: 14 February 2008/Published online: 14 March 2008

(C) Springer Science+Business Media, LLC 2008

\begin{abstract}
Recent functional magnetic resonance imaging (fMRI) and event-related brain potential (ERP) studies provide empirical support for the notion that emotional cues guide selective attention. Extending this line of research, whole head magneto-encephalogram (MEG) was measured while participants viewed in separate experimental blocks a continuous stream of either pleasant and neutral or unpleasant and neutral pictures, presented for $330 \mathrm{~ms}$ each. Event-related magnetic fields (ERF) were analyzed after intersubject sensor coregistration, complemented by minimum norm estimates (MNE) to explore neural generator sources. Both streams of analysis converge by demonstrating the selective emotion processing in an early (120-170 ms) and a late time interval (220$310 \mathrm{~ms}$ ). ERF analysis revealed that the polarity of the emotion difference fields was reversed across early and late intervals suggesting distinct patterns of activation in the visual processing stream. Source analysis revealed the amplified processing of emotional pictures in visual processing areas with more pronounced occipito-parietotemporal activation in the early time interval, and a stronger engagement of more anterior, temporal, regions in the later interval. Confirming previous ERP studies
\end{abstract}

P. Peyk (

Department of Psychology, University of Basel, Missionsstrasse 60/62, 4055 Basel, Switzerland

e-mail: peter.peyk@unibas.ch

H. T. Schupp · T. Elbert

Department of Psychology, University of Konstanz, Konstanz, Germany

M. Junghöfer

Institute for Biomagnetism and Biosignal Analysis, University of Münster, Muenster, Germany showing facilitated emotion processing, the present data suggest that MEG provides a complementary look at the spread of activation in the visual processing stream.

Keywords Emotion - Attention - MEG - ERF . ERP · Early posterior negativity

\section{Introduction}

Pictures varying in hedonic valence and emotional arousal have been successfully used to explore emotional processing across response channels. Specifically, differences in autonomic (electrodermal activity, heart rate), reflex (startle blink), and somatic (facial electromyography) measures are reliably elicited by these images (reviewed in ref. [2, 12]). In recent years, functional magnetic resonance imaging (fMRI) and event-related brain potentials (ERP) served to explore the neural mechanisms of emotional perception and evaluation. Building upon this line of research, the present study utilized whole head magnetoencephalography (MEG) to explore the temporal dynamics of emotional stimulus processing.

According to a motivational model of emotion [22, 23], pleasant and unpleasant stimuli engage appetitive and aversive motivational systems, which are important for implementing actions to stimuli that can sustain and threaten the life of the organism. The view that emotion is in part organized by underlying motivational factors is supported by research utilizing verbal reports, which consistently demonstrates the primacy of the valence dimension. Furthermore, both motivational subsystems can vary in terms of engagement or activation reflecting the arousal level, which is reliably observed as second dimension in studies of natural language and verbal reports 
$[22,23]$. While emotions are primarily considered as action sets, which prepare the organism for either avoidance or approach related actions, such a perspective also suggests that emotional cues direct attentional resources at the evaluative and perceptual level. Specifically, efficient preparation and organization of appropriate behavioral responses require a rapid extraction of critical information from the environment. In this respect, emotional cues direct attentional resources [23, 28].

The hypothesis that emotional cues guide selective visual attention and receive enhanced processing is supported by neuroimaging studies. For instance, functional Magnetic Resonance Imaging (fMRI) reliably revealed increased BOLD (Blood Oxygen Level Dependent) signals in associative visual regions (extrastriate, occipito-parietal, and inferior temporal cortex) and subcortical limbic structures when viewing emotionally arousing compared to neutral pictures [3, 19, 32]. It has been suggested that the enhanced perceptual processing at the cortical level is regulated by activity in subcortical limbic structures [36]. Furthermore, a series of recent event-related potential studies detailed the temporal dynamics of selective emotion processing during visual perception [33]. Distinguishing distinct cognitive subsystems of stimulus recognition, selective emotion processing is already observed during initial stimulus identification preceding the conscious awareness and elaborate processing of emotional cues [27, 33]. Specifically, it was consistently found that emotional compared to neutral pictures are associated with a relative early posterior negativity (EPN) over temporo-occipital sensor regions developing around $120-150 \mathrm{~ms}$ after stimulus onset and lasting for 150-200 ms [10, 15, 29]. As found in fMRI studies, the EPN modulation was obtained for pleasant and unpleasant, compared to neutral, pictures and most pronounced for emotional stimuli rated high in emotional arousal [33].

The present study used evoked related magnetic field (ERF) measurements to investigate the processing of emotional cues. As in previous research [15]; the rapid picture presentation technique has been chosen to reveal the brain's capacity of emotion discrimination under conditions of high perceptual load and conceptual masking. In separate blocks, pleasant and unpleasant IAPS pictures were presented as rapid serial stream $(3 \mathrm{~Hz})$ alternating with neutral images $[15,29]$. The main goal of the present study was to determine the magnetic counterpart of the EPN (EPN-M). Beyond the replication of previous findings with another technique to measure brain activity, MEG measurements provide several methodological advantages compared to EEG recordings. Magnetic compared to electrical field topographies are less influenced by volume conductor properties (e.g., scalp thickness) resulting in more robust estimates of neural activity by inverse source modeling. Furthermore, MEG measures are reference free circumventing the problems associated with the selection of the 'best' reference montage in ERP studies [18]. In order to compare ERF emotion effects to ERP recordings obtained in previous studies, a MEG sensor standardization technique was applied to allow the calculation of grand mean MEG waveforms in the sensor space [18]. A secondary objective of the study was to capitalize on the differential sensitivity of MEG and EEG recordings regarding radially and tangentially oriented generator structures. Specifically, while EEG is sensitive to both radial and tangentially oriented generator structures the MEG is almost only sensitive to tangential but almost blind to radial oriented neural sources. Thus, MEG measurements promised to reveal more specific patterns of brain activity within the first three hundred milliseconds of perceptual processing.

\section{Methods}

\section{Participants}

Participants were 26 (13 females) introductory psychology students from the University of Konstanz. Participants were between the ages of 19 and 26 years $(M=22.8)$. The participants provided written informed consent for the protocol approved by the Review Board of the University of Konstanz.

\section{Stimulus Materials and Procedure}

High-arousing pleasant $(N=100)$, high-arousing unpleasant $(N=100)$, and neutral pictures $(N=100)$ were selected from the International Affective Picture System (IAPS) [21]. Statistical tests revealed that physical picture parameters such as brightness, contrast, color distribution, physical complexity or spatial frequencies did not differ across the picture categories. Additionally the relation of simple figure/ ground versus complex scene stimuli did not differ significantly between the picture groups [4]. The experiment consisted of two experimental blocks lasting approximately $67 \mathrm{~s}$ each. In each condition, a continuous stream of pictures was shown with each picture presented for $330 \mathrm{~ms}$. In one block, 200 pleasant and neutral pictures were presented, while the other block contained a stream of 200 unpleasant and neutral pictures. Block order was balanced across participants. In each block, emotionally arousing and neutral stimulus contents were presented in an alternating sequence. This procedure was chosen to maximize the EPN as correlate of affective modulation because a neutral picture preceding an emotional cue has comparatively less detrimental effect on the posterior negativity elicited by the subsequent picture compared to an emotional cue preceding a neutral image [10]. To minimize ocular artifacts, participants were asked to 
passively view the stimuli while keeping their eyes focused on a small central fixation cross, which was overlaid on the pictures.

\section{Apparatus and Data Analysis}

Magnetic fields were measured with a 148-channel whole head magnetometer (MAGNES 2500 WH, 4D Neuroimages, San Diego, USA) using a sampling rate of $678.17 \mathrm{~Hz}$. Data were recorded continuously applying band-pass filtering from 0.1 to $200 \mathrm{~Hz}$. MEG analysis were conducted with the Matlab-based EMEGS software [17] (www.emegs.org). The method for statistical control of artifacts was used for data editing and artifact rejection [16]. This procedure (1) detects individual channel artifacts, (2) detects global artifacts, (3) replaces artifact-contaminated sensors with spline interpolation statistically weighted on the basis of all remaining sensors, and (4) computes the variance of the signal across trials to document the stability of the averaged waveform. The rejection of artifact-contaminated trials and sensor epochs relies on the calculation of statistical parameters for the absolute measured magnetic field amplitudes over time, their standard deviation over time, the maximum of their gradient over time (first temporal derivative), and the determination of boundaries for each of these three parameters.

Finally, average event-related magnetic fields were calculated for each picture category, individual sensor, and subject, respectively. Baseline correction was calculated from 330 to $330 \mathrm{~ms}$ after stimulus onset, thus comprising the duration of one stimulus of both categories. In this way, baseline correction was identical for both affective conditions in each session, allowing an optimal analysis of the category differences.

\section{ERF Analysis}

First, variance due to differential positioning of the individual subjects head in the MEG scanner has been minimized by a sensor standardization procedure [18, 26]. With this standardization technique, the event related magnetic field for each participant is estimated as if it would have been measured with a standardized sensor configuration identical for all participants in the scanner.

For statistical analysis, a two-step procedure used in previous ERP research was applied to analyze the modulation of the MEG waveform as a function of affect. First, $t$-tests were calculated for each time point after picture onset separately for each individual MEG sensor in order to identify the temporal and spatial modulation of the ERF as a function of emotionality. Specifically, a first analysis contrasted pleasant with neutral pictures while a second analysis included unpleasant and neutral picture materials.
These waveform analyses were conducted using a significance criterion of $P<0.01$. In order to avoid false positives, significant effects were only considered meaningful, when the effects were observed for at least eight continuous data points $(32 \mathrm{~ms})$ and two neighboring sensors revealing significant affective modulation. As detailed in the result section an early (120-170 ms) and a late interval (220-310 ms) appeared to show the dominant effects of emotion in the pointwise ERF analysis.

To more precisely determine statistical effects associated with picture emotionality, this outcome of the single sensor waveform analysis was followed-up by conventional ANOVAs: for the early component (120-170 ms), the ERF amplitude was scored as mean activity across this window and over one left temporal and one right temporal sensor cluster with 5 sensors per cluster as shown in the upper row of Fig. 1 (left: '4d neuroimage' sensors 135, 117, 116, 134, 133; right: 127, 109, 108, $110,126)$; for the late component (220-310 ms), the ERF were scored as mean activities across this time window and over larger, more widespread sensor groups with 13 sensors per cluster (left: 79, 98, 99, 100, 115, 116, 117, 118, 132, 133, 134, 135, 136; right: 109, 125, 126, 127, 143, 144, 145, 146, 147, 128, 107, 108, 87). Each sensor cluster was analyzed separately in a two-factorial repeated measures ANOVA including the factors AROUSAL (emotional vs. neutral) and VALENCE (pleasant vs. unpleasant), thus comprising both conditions from each experimental block.

\section{L2-Minimum-Norm Analyses}

The L2-Minimum-Norm-Pseudoinverse (L2MNP) is an inverse modeling technique, which estimates cortical generator structures without any a priori assumptions regarding the location and/or number of current sources [1, 11]. In addition, the L2MNP determines only that part of all possible sources which is solely determined by the measured magnetic fields.

As source model we used a spherical shell with evenly distributed 2 (azimutal and polar direction, radial dipoles do not generate magnetic fields outside of a sphere) $\times 360$ dipoles. A source shell radius of $87 \%$ of the individually fitted head radius has been chosen, roughly corresponding to the grey matter volume. Across, all participants and conditions, a Tikhonov regularization parameter $\lambda$ of 0.02 was applied.

Topographies of dipole direction independent neural activities - the vector length of the generator activities at each position-were calculated for each individual subject, condition and time point based on the averaged magnetic field distributions and the individual sensor positions for each subject and run. 
Fig. 1 (Top) Waveforms of mean ERF regional amplitudes evoked by emotional and neutral pictures for a left temporal and right temporal sensor cluster (lower right edge of the subfigures). (Middle) 3Hz-highpass filtered waveforms of mean ERF regional amplitudes as in the top row. Condition differences before $100 \mathrm{~ms}$ are erased by the filter while early and late EPN-M effects remain visible. (Bottom) Time course of corresponding neural generator activity as estimated by L2-Minimum-Norm inverse modeling within left and right hemispheric temporo-occipital regions of interest (lower right edge of the subfigures)
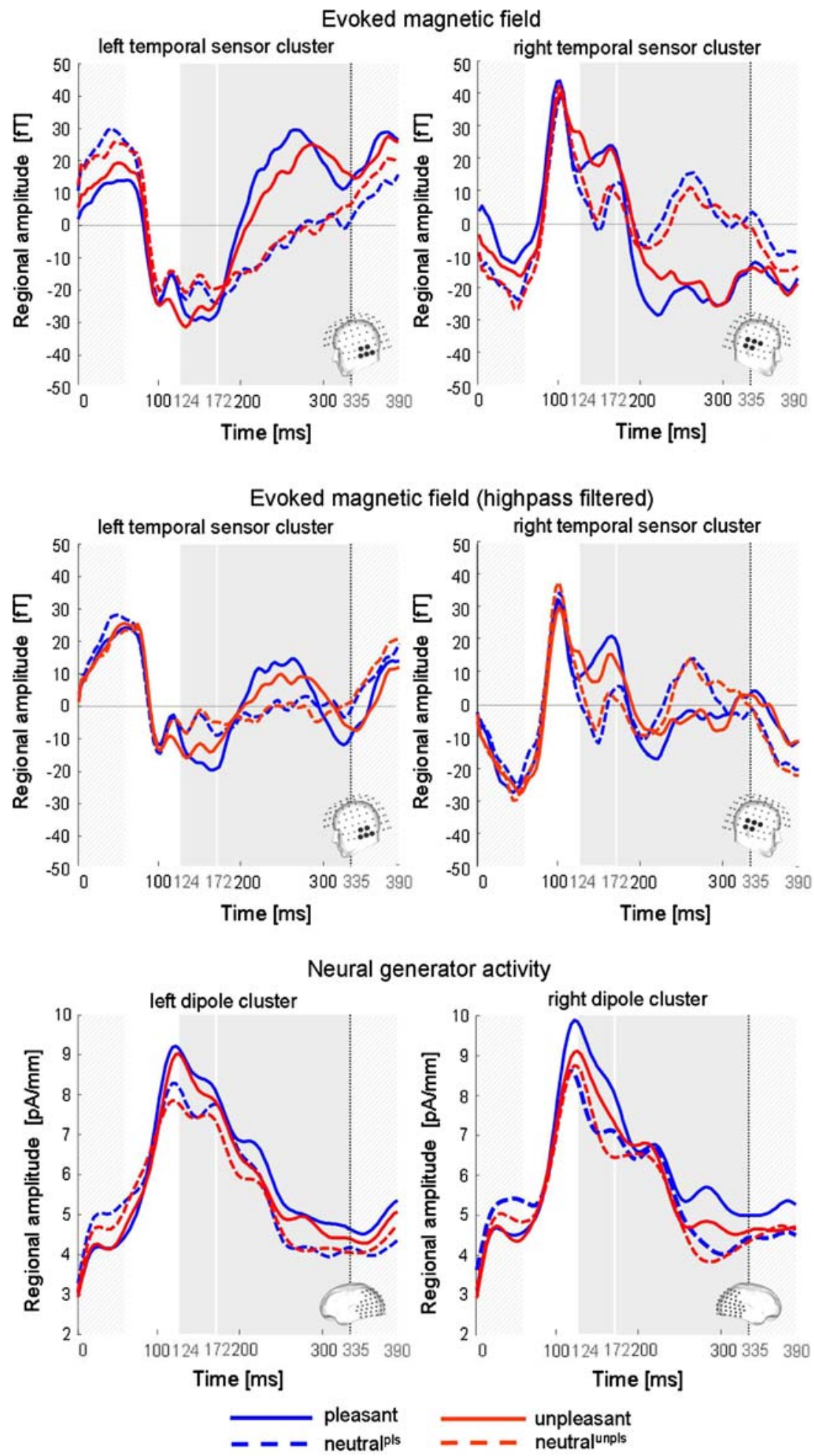

For statistical analysis of these neuronal generator activities, a similar approach as for the magnetic fields was applied: First, $t$-tests were calculated for each time point after picture onset separately for each individual dipole to identify the temporal and spatial modulation of the ERP as a function of emotionality, again separately for pleasant vs. neutral and unpleasant vs. neutral pictures. These waveform analyses were conducted using a significance criterion of $P<0.05$ and effects were only considered meaningful when observed for at least eight continuous data points $(32 \mathrm{~ms})$.

As for the magnetic fields, the single dipole waveform analyses were followed by conventional ANOVAs: for the 
early component, the dipole activities were scored as mean activity over two symmetric occipito-parietal dipole groups with 37 dipoles per group over the time interval from 120 to $170 \mathrm{~ms}$; for the late component, dipole activities were scored as mean activity over two larger occipito-temporal dipole groups with 54 dipoles per group over a time interval from 220 to $310 \mathrm{~ms}$. The early and late components were analyzed separately in three factorial repeated measures ANOVAs including the factors AROUSAL (emotional vs. neutral), VALENCE (pleasant vs. unpleasant) and LATERALITY (left vs. right). When appropriate, significant effects were followed up by Bonferroni corrected post-hoc contrasts.

\section{Results}

\section{Waveform Analyses}

The upper row of Fig. 1 shows the ERF regional amplitudes for the left temporal and right temporal sensor cluster used also for the statistical analysis of the early ERF component (described above). ERF waveforms presented a strong M100 component, with negative (ingoing) magnetic field amplitudes at sensors located over left temporal regions and corresponding positive (outgoing) magnetic field amplitudes over right temporal regions. While the ERFs of the neutral conditions approached the zero line, ERFs of the emotional conditions showed (i) a prolonged M100 corresponding the first analyzed interval from 120 to $170 \mathrm{~ms}$, followed by (ii) a characteristic polarity reversal corresponding to the second analyzed interval from 220 to $310 \mathrm{~ms}$. The lower part of Fig. 1 shows the regional estimated generator activities for the left posterior and right posterior dipole cluster (to best illustrate both early and late component effects, the used dipole clusters for the graph represent a set which was slightly different from the clusters used for statistical analysis of the early and late component).

The topography of the ERF and estimated neural generator activity is further illustrated in Figs. 2 and 3, complemented by statistical findings of the single sensor waveform analyses. ERF and neural source estimation analyses provide converging evidence for the selective emotion processing in an early and late time interval. Please note that ERF topographies evince polarity reversals, both with respect to hemisphere and differential emotion processing, while the neural source estimates suggest preferential emotion processing for both time intervals.

\section{ERF Component Analysis}

Figure 4 (upper rows) shows the mean amplitudes of the early and late ERF components as a function of picture category and laterality. Statistical analysis revealed a highly significant effect of AROUSAL for the early component (120-170 ms) at left temporal and right temporal sensors (left: $F(1,25)=11.3, P<0.01$; right: $F(1,25)=27.3$, $P<0.0001$ ), indicating larger negative (left) and positive (right) field amplitudes for the emotional picture categories compared to the neutral ones. The hedonic valence of the pictures did not influence the magnetic fields (VALENCE left: $F(1,25)=0.5$, ns; right: $F(1,25)=0.9$; ns), nor was there a significant interaction of valence and arousal (left: $F(1,25)=0.4$, ns; right: $F(1,25)=0.7, \mathrm{~ns})$.

Arousal dependant ERF differences during the later window (220-310 ms) were again highly significant (AROUSAL left: $F(1,25)=43.6, P<0.0001$; right: $F(1,25)=33.5$, $P<0.0001$ ), yet of opposite polarity with larger positive amplitudes over left temporal and larger negative amplitudes over right temporal areas compared to the early effect. No main effect of valence or interaction of valence and arousal was observed (left: VALENCE: $F(1,25)=0.8$, ns; VALENCE $\times$ AROUSAL: $F(1,25)=0.9$, ns; right: VALENCE: $F(1,25)=0.1, \mathrm{~ns}$; VALENCE $\times$ AROUSAL: $F(1,25)=2.5, \mathrm{~ns})$.

\section{L2-Minimum-Norm Component Analysis}

Estimated neural generator activities showed a similar pattern as the magnetic fields. Mean dipole activity is shown in Fig. 4 (lower rows) of the early and late interval as a function of picture category and laterality. For the early component, the factor AROUSAL was highly significant $(F(1,25)=28.1$, $P<0.0001$ ), equally pronounced for left and right dipoles, as indicated by the missing interaction with LATERALITY (AROUSAL $\times$ LATERALITY: $F(1,25)=2.0$, ns). The only other significant effect was an AROUSAL $\times$ VALENCE interaction $(F(1,25)=4.72 ; P<0.05)$, caused by larger activities for the pleasant than the unpleasant condition (pleasant vs. unpleasant: $P<0.05$ ). Pairwise comparisons of both emotional categories with their neutral counterparts were significant (pleasant vs. neutral ${ }_{\mathrm{pls}}: P<$ 0.0001 ; unpleasant vs. neutral unpls $: P<0.0001$ ), while the

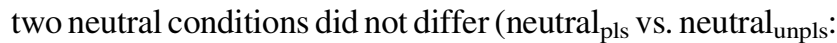
$P=0.99$ ).

Source strengths of the late component showed a similar pattern, with a significant main effect of AROUSAL $(F(1,25)=13.6, P<0.001)$, no lateralization (LATERALITY: $F(1,25)=0.17, \mathrm{~ns})$ and a significant AROUSAL $\times$ VALENCE interaction $(F(1,25)=6.9, P<0.01)$, due to larger amplitudes of the pleasant condition (pleasant vs. unpleasant: $P<0.05)$. Again, contrasts with neutral pictures were significant (pleasant vs. neutral ${ }_{\mathrm{pls}}: P<0.0001$; unpleasant vs. neutral unpls: $P<0.05$ ) whilst the neutral conditions evoked similar generator activities (neutral ${ }_{\mathrm{pls}}$ vs. neutral $\mathrm{unpls}_{\mathrm{u}}$ : $P=0.99)$. 
Fig. 2 Collapsing across meaningful time bins, topographies of ERF differences (contour line plots) and corresponding statistical parametric maps of uncorrected ERF $t$-statistics (red-grey-blue plots) for emotional versus neutral picture processing are shown. Model heads illustrate left (lower two rows) and right (upper two rows) views. $T$-values of \pm 1.7 correspond $\alpha=0.05$
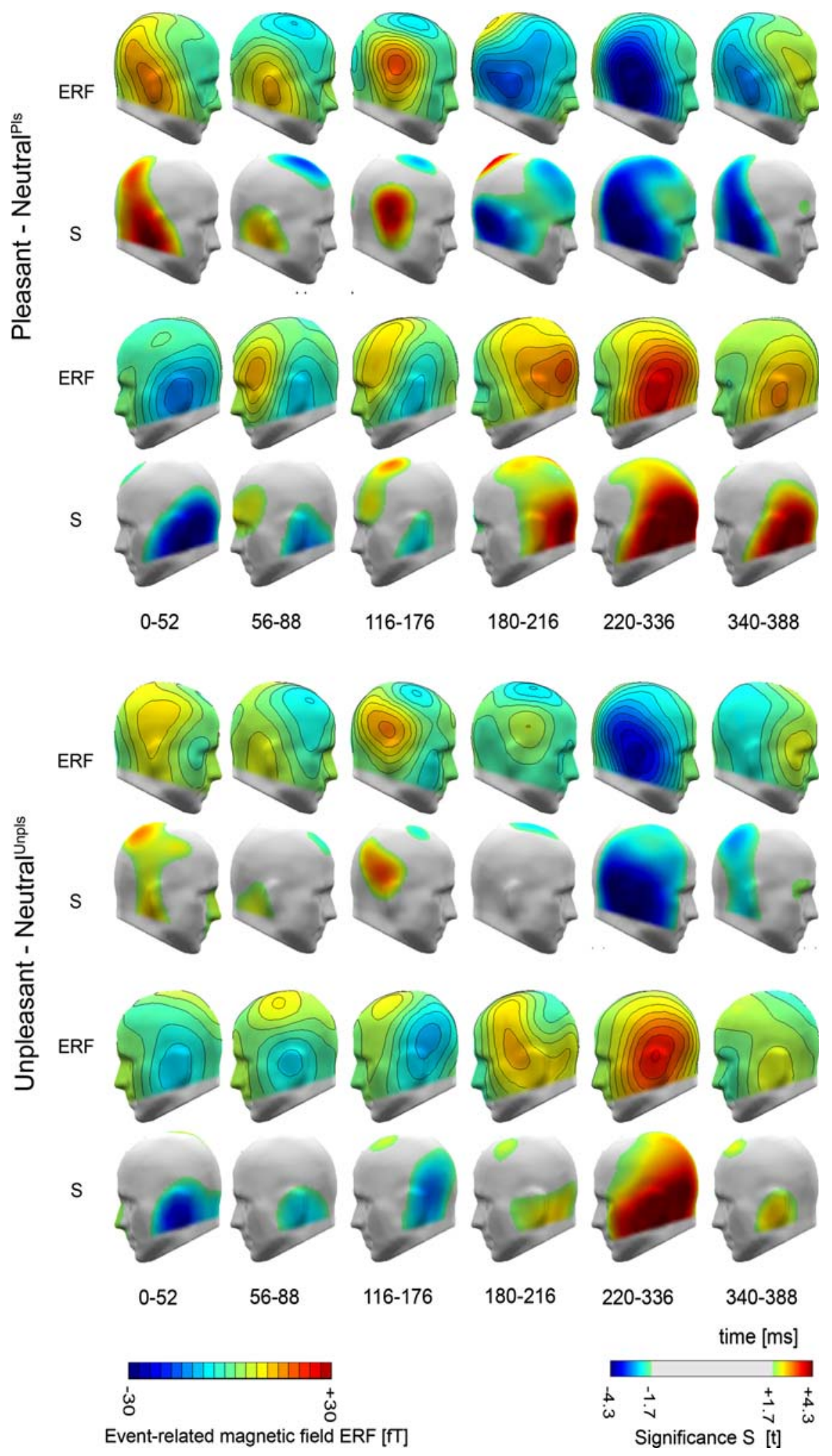

\section{Control Analyses}

Inspection of the ERF waveforms and topographies (Figs. $1,2)$ reveals the continued processing effects of the first picture in a picture pair, which precedes the P100-M component elicited by the following picture. This continuation of the differential processing of emotional pictures is highlighted in Fig. 1 (top row) by showing an additional time interval from $335 \mathrm{~ms}$ up to $390 \mathrm{~ms}$ (i.e., 5-55 ms of the subsequent picture pair). Emotional compared to 
Fig. 3 Topographies of estimated generator activity differences (contour line plots) and corresponding statistical parametric maps of uncorrected ERF $t$-statistics (red-grey-blue plots) for emotional versus neutral picture processing at consecutive time intervals of interest. Model heads illustrate left (lower two rows) and right (upper two rows) views. $T$-values of \pm 1.7 correspond $\alpha=0.05$

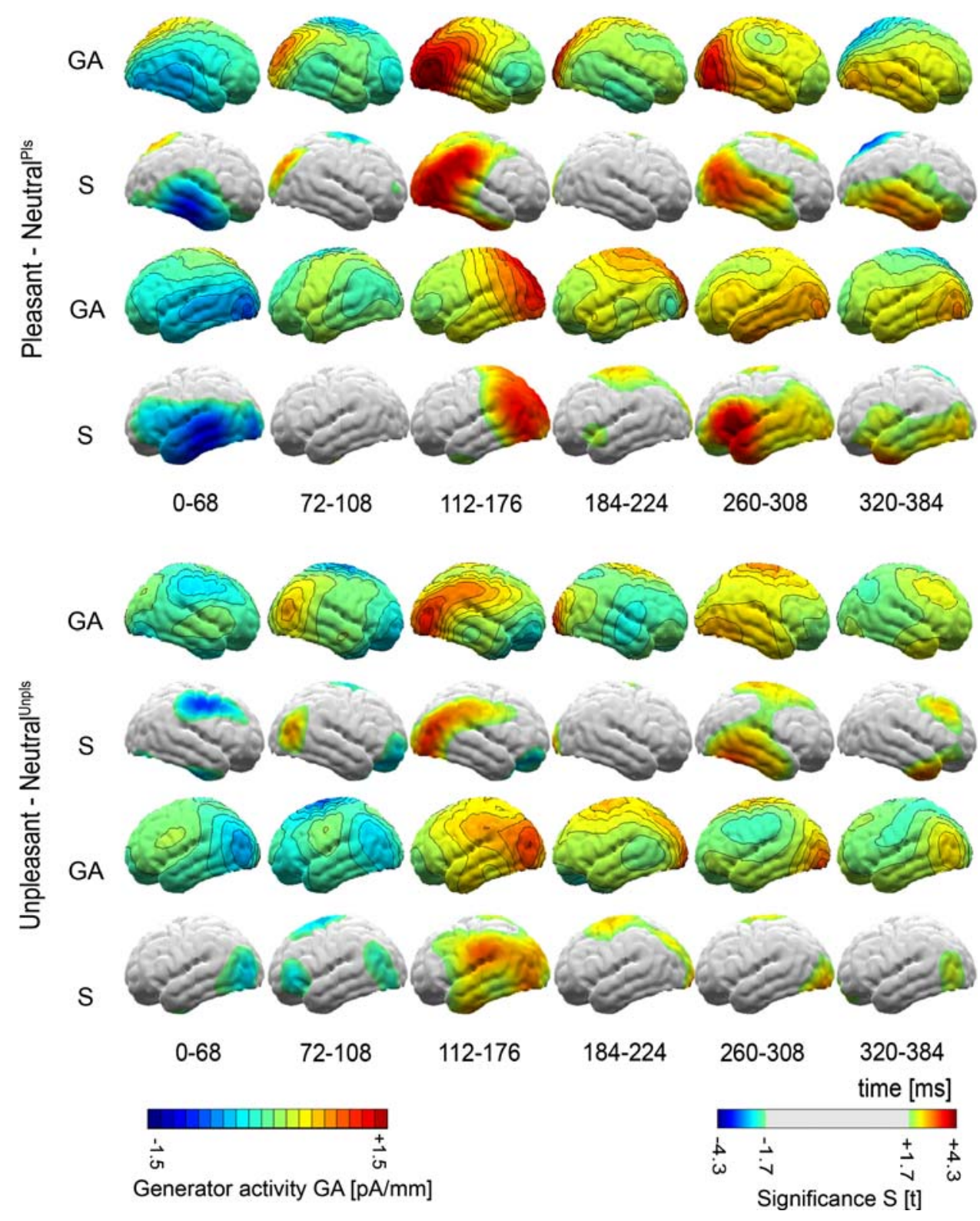

neutral pictures evince a relative positive field shift over left temporal sites and the effect reverses polarity over right temporal sites. An according effect can be discerned when inspecting early processing period from 0 to $100 \mathrm{~ms}$. Due to the alternating presentation of emotional and neutral pictures, this effect is of opposite polarity. Furthermore, as expected, inspection of Fig. 2 demonstrates a highly similar topography of the early (0-52 ms) and the continued differential emotional effect (340-388 ms). Of more interest, inspection of the time window from 56 to $88 \mathrm{~ms}$ shows that continued effects associated with the preceding picture processing decrease in amplitude. In contrast, in the 116-176 ms time interval, differentiation between emotional and neutral pictures are pronounced and with a different topography suggesting that these effects are no longer a continuation of the preceding picture processing but reflect early differential emotional processing of the actual picture pair. The time courses and topographies of the corresponding estimated neural generators (L2MNP) further support the notion of slowly decreasing residual activity prior the P100-M but newly appearing differences of emotional processing after $120 \mathrm{~ms}$ : While emotional compared to neutral pictures evoke amplified processing in the early (120-170 ms) and late (220-310 ms) time intervals, differences appear inverted-since belonging to the preceding picture pair-and continuously decreasing within the time window preceding the P100-M.

Applying a $3 \mathrm{~Hz}$ temporal highpass filter to the event related magnetic field data provided an additional control analysis that the effects of emotionality in the early time 
Fig. 4 Box plots for the Arousal (2) $\times$ Valence (2) ANOVAs for the early (120$170 \mathrm{~ms})$ and the late $(220$ $310 \mathrm{~ms}$ ) time interval with mean ERF data at left and right temporal sensor clusters (top) as well as mean estimated neural activities at left and right posterior regions of interest (sensor cluster and regions shown in Fig. 1)
ERF 124-168 ms
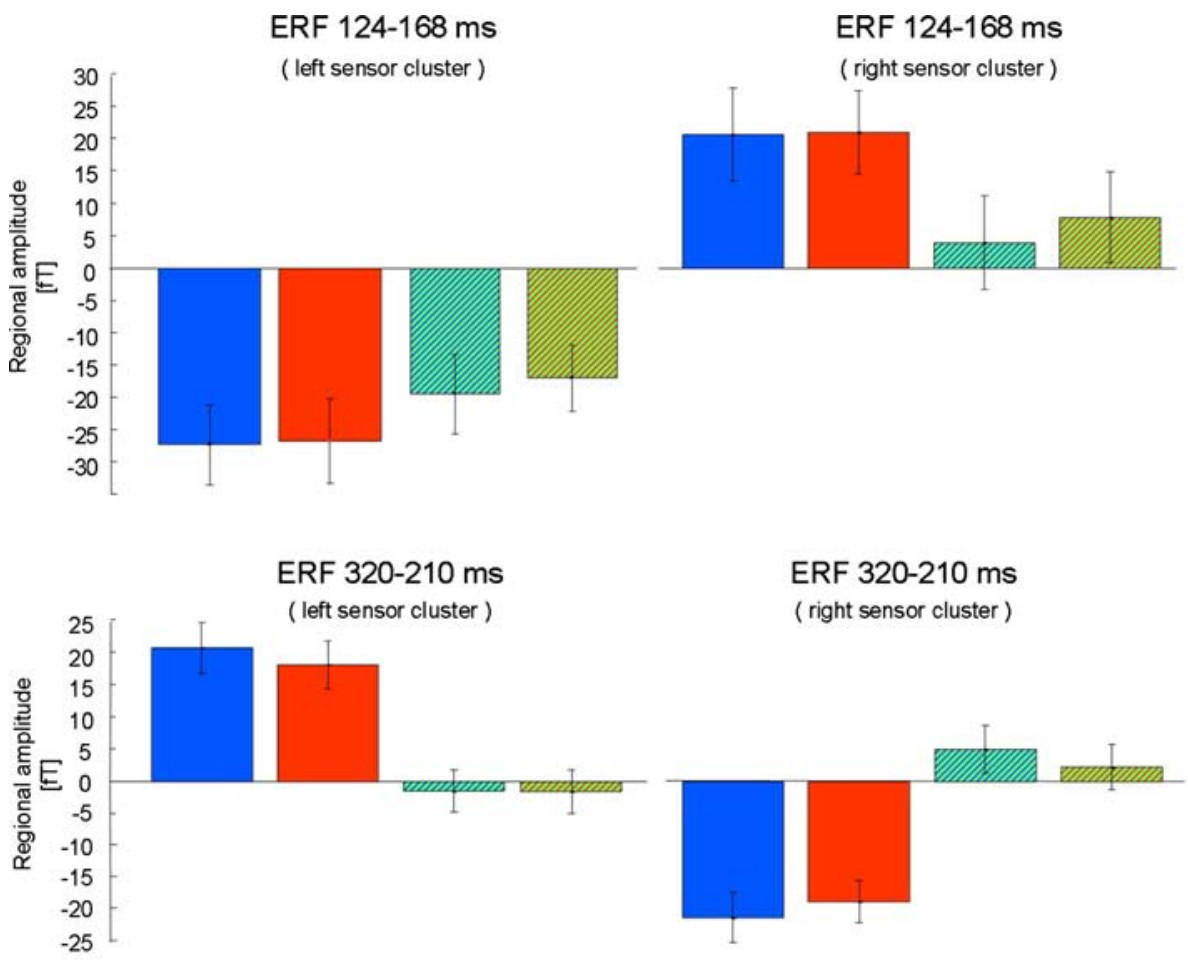

Neural generator activity $124-168 \mathrm{~ms}$ (left posterior dipole cluster)

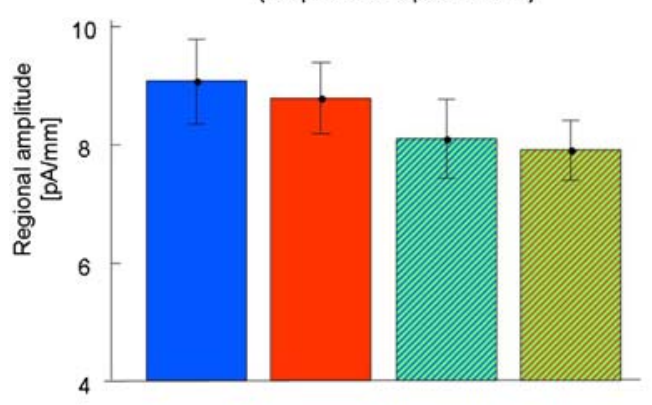

Neural generator activity $220-310$ ms
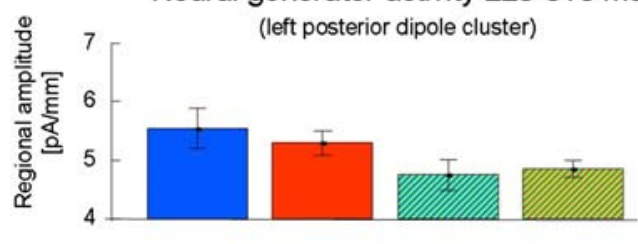

pleasant
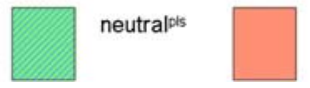

unpleasant

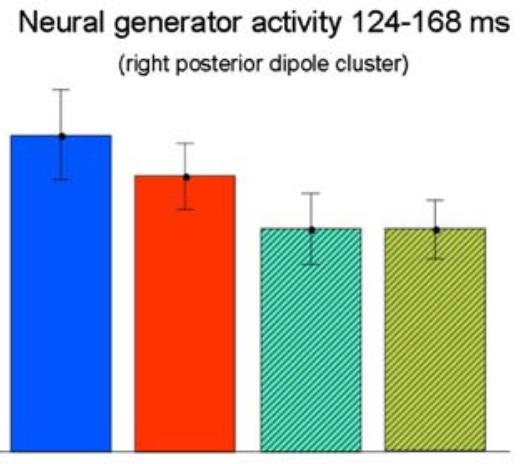

ERF $320-210 \mathrm{~ms}$

( right sensor cluster)

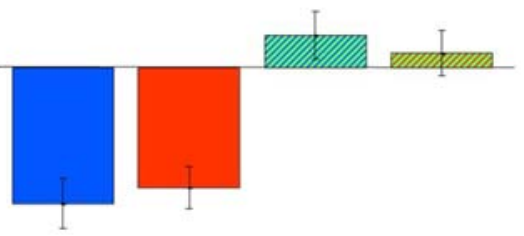

Neural generator activity $220-310$ ms (right posterior dipole cluster)
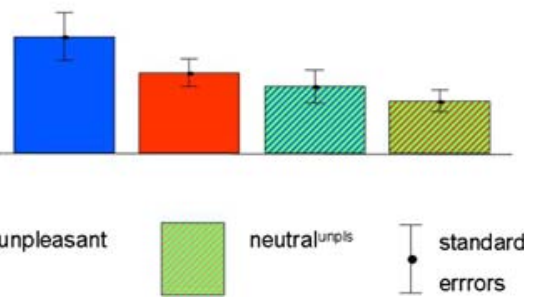

interval (120-170 ms) are not merely a consequence of continued differential processing. This highpass filter strongly attenuates sustained effects such as the slowly decreasing residual processing while focusing on shortterm and rapid changes of neural activity. As a result, this analysis amounts to a baseline independent investigation of ERF effects. As shown in Fig. 5, the highpass filtering completely erased the effects of continued processing in the $0-116$ ms time interval while ERF difference effects in the early and late EPN-M time intervals were pronounced and significant. Overall, these control analysis provide good evidence that effects appearing in the time window from 120 to $170 \mathrm{~ms}$ reflect early differential emotional processing of the actual picture pair while effects preceding 


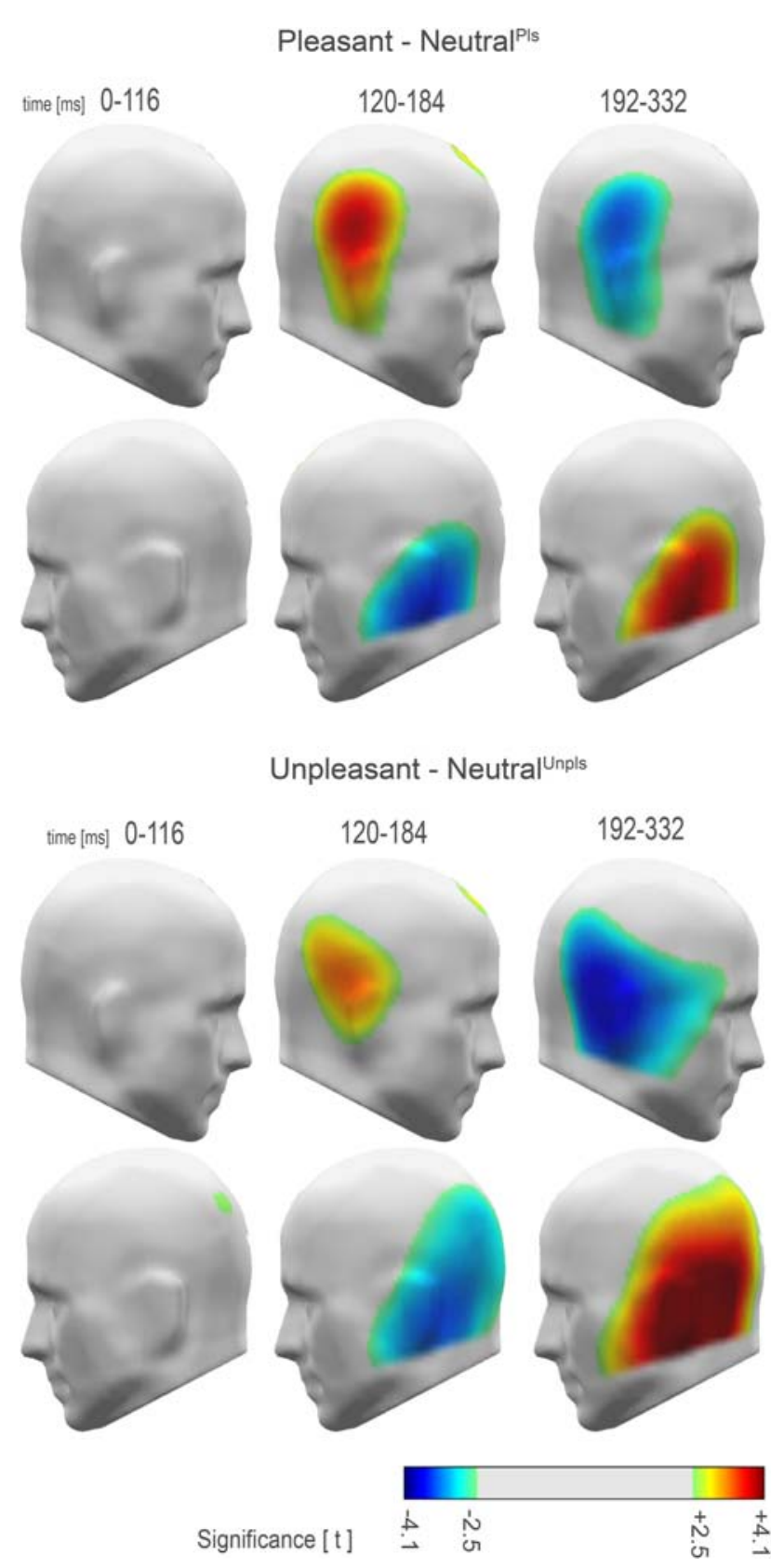

Fig. 5 Statistical parametric maps of uncorrected ERF $t$-statistics at three time intervals of interest after application of a $3 \mathrm{~Hz}$ highpass filter. $T$-values of \pm 2.5 correspond $\alpha=0.01$

the P100-M reflect effects continued effects of the preceding picture pair.

\section{Discussion}

The present data show a strong and reliable magnetic counterpart to the EPN reported in ERP studies with strongly amplified processing of both, unpleasant and pleasant emotional pictures compared to neutral material starting around
$120 \mathrm{~ms}$ after stimulus onset. The difference fields were maximally pronounced at occipito-temporal sensor clusters appearing in an early (120-170 ms) and a later time interval (220-310 ms). As typical for MEG measures, magnetic fields evoked by emotional and neutral pictures (and their difference fields) revealed a polarity reversal between left and right sensor clusters when neural structures with similar dipole orientation in both hemispheres are activated. Interestingly, selective emotion processing in the early and later time interval was of opposite polarity-presumably reflecting the spread of activation in occipito-parieto-temporal structures. Thus, while the preferential emotion processing observed in previous EEG studies was confirmed, MEG allowed a complementary look at the neuronal processes involved in this modulation.

The described biphasic modulation concurs with recent findings. For instance [7], reported a similar two-component pattern for differential processing of nude pictures compared to neutral pictorial stimuli. Studying a broader array of picture contents (erotic couples, sports, household objects, threat related pictures and mutilations) also revealed an early (following the P100-M) and a later component (after $200 \mathrm{~ms}$ ) differentiating emotional from neutral contents [5]. Similar observations were made in a further study presenting IAPS pictures in a clinical sample [31]. Similar to MEG studies of natural scenes, a biphasic pattern of activation is also observed in studies exploring emotional processing of human faces [8, 14, 24, 25, 30, 35] although activation in the later time window is usually weaker compared to the early interval and sometimes not discussed. Taken together, it appears that MEG consistently shows a different pattern of modulation than the EEG in the time interval from 120 to 300 .

The opposite polarity of the emotion difference in early and late time intervals can be assumed to reflect distinct states of activation in the visual processing stream. In contrast, electrical field recordings have not allowed this differentiation, revealing rather a uniform relative negative potential shift when comparing emotional and neutral picture contents, developing around $120-150 \mathrm{~ms}$ and sustained until 300-350 ms [10, 15, 29]. Similar uniform modulations have been observed when studying emotional faces [34] and emotional word material [13, 20].

Source analyses of the emotional modulation in the early and late time interval suggests increased activation in posterior brain regions for both time intervals. As expected, activation in anterior temporal regions was more pronounced in the later time interval, while occipito-parieto-temporal activations were more apparent in the earlier time interval presumably reflecting the spread of activation in visual processing. However, the most straightforward approach to disambiguate the differences among early and late emotion processing seems to be based on the magnetic 
field waveforms which reveal polarity reversal of emotion difference effects. For these reasons, the application of a sensor standardization procedure seems highly advantageous $[18,26]$, which enables the calculation of grand mean ERFs by correcting for individual differences in sensor positioning in the MEG scanner.

A noteworthy finding is the breakdown of difference activation for emotional compared to neutral picture processing in the time window from 180 to $220 \mathrm{~ms}$. The interruption of selective emotion processing in this time interval seems unlikely when considering the ERP studies. Specifically, increased activation in occipito-parieto-temproal structures are brought out by source analysis in electrical field recordings in this time interval [15]. Accordingly, it is suggested that the breakdown of differential emotion processing represents the zero crossing-the destructive interference of contrary magnetic field polarity-of the averaged generated magnetic fields with the spread of activation along the visual pathway. Future studies with simultaneous recordings of EEG and MEG may provide more direct evidence for this hypothesis [9].

The primary aim of the present study was to reveal the differentiation among emotional compared to neutral pictures. The present study therefore utilized separate blocks in which pleasant and unpleasant contents were compared with neutral pictures. While the predominant modulation in the early and late time interval was similarly observed for unpleasant and pleasant stimuli, differences among pleasant and unpleasant pictures were detected as well, although considerably smaller in effect sizes. More specifically, a stronger and earlier onset of difference processing for unpleasant compared to pleasant pictures in the early time interval and a stronger and earlier onset of difference processing for pleasant material in the later time interval was found [6]. These findings suggest differences for appetitive and aversive picture contents, which await exploration in future studies.

The present study extends the accumulating evidence for the prioritized processing of emotional pictures early in the visual processing stream. ERP studies consistently revealed a relative negative potential over posterior sensor sites associated with emotional stimulus processing in a time interval from approximately $120-350 \mathrm{~ms}$. Beyond confirming ERP findings of differential brain activity to emotional stimuli, the assessment of evoked magnetic fields determined two distinct patterns of brain activity in early stimulus processing. Anticipating research to come, this study sets the stage for increased precision in investigating the emotional attention capture in the visual processing stream.

Acknowledgments This research was supported by Grants from the German research foundation JU-445-4-1 and Schu1074/11-1 to Markus Junghöfer and Harald Schupp.

\section{References}

1. Baillet S, Mosher JC, Leahy RM. Electromagnetic brain mapping. IEEE Signal Process Mag 2001;18(66):14-30.

2. Bradley MM. Emotion and motivation. In: Cacioppo JT, Tassinary LG, Berntson G, editors. Handbook of psychophysiology. New York: Cambridge University Press; 2000. p. 602-642.

3. Bradley MM, Sabatinelli D, Lang PJ, Fitzsimmons JR, King W, Desai P. Activation of the visual cortex in motivated attention. Behav Neurosci 2003;117(2):369-80.

4. Bradley MM, Hamby S, Löw A, Lang PJ. Brain potentials in perception: picture complexity and emotional arousal. Psychophysiology 2007;44(3):364-73.

5. Buodo G, Peyk P, Junghöfer M, Palomba D, Rockstroh B. Electromagnetic indication of hypervigilant responses to emotional stimuli in blood-injection-injury fear. Neurosci Lett 2007;424(2):100-5.

6. Codispoti M, Ferrari V, Bradley MM. Repetition and eventrelated potentials: distinguishing early and late processes in affective picture perception. J Cognitive Neurosci 2007;19: 577-86.

7. Costa M, Braun C, Birbaumer N. Gender differences in response to pictures of nudes: a magnetoencephalographic study. Biol Psychol 2003;63(2):129-47.

8. Dolan RJ, Heinze HJ, Hurlemann R, Hinrichs H. Magnetoencephalography (MEG) determined temporal modulation of visual and auditory sensory processing in the context of classical conditioning to faces. Neuroimage 2006;32(2):778-89.

9. Eulitz C, Eulitz H, Elbert T. Differential outcomes from magneto- and electroencephalography for the analysis of human cognition. Neurosci Lett 1997;227(3):185-8.

10. Flaisch T, Junghöfer M, Bradley MM, Schupp HT, Lang PJ. Rapid picture processing: affective primes and targets. Psychophysiology 2008;45(1):1-10.

11. Hamalainen MS, Ilmoniemi RJ. Interpreting magnetic fields of the brain: minimum norm estimates. Med Biol Eng Comput 1994;32(1):35-42.

12. Hamm AO, Weike AI, Schupp HT, Treig T, Dressel A, Kessler C. Affective blindsight: intact fear conditioning to a visual cue in a cortically blind patient. Brain 2003;126(Pt 2):267-75.

13. Herbert C, Junghöfer M, Kissler J. Event related potentials to emotional adjectives during reading. Psychophysiology (in press).

14. Ishai A, Bikle PC, Ungerleider LG. Temporal dynamics of face repetition suppression. Brain Res Bull 2006;70(4-6): 289-95.

15. Junghöfer M, Bradley MM, Elbert T, Lang PJ. Fleeting images: a new look at early emotion discrimination. Psychophysiology 2001;38(2):175-8.

16. Junghöfer M, Elbert T, Tucker DM, Rockstroh B. Statistical control of artifacts in dense array EEG/MEG studies. Psychophysiology 2000;37(4):523-32.

17. Junghöfer M, Peyk P. Analysis of electrical potentials and magnetic fields of the brain. Matlab Select 2004;2:24-8. EMEGS software is freely available at http://www.emegs.org.

18. Junghöfer M, Peyk P, Flaisch T, Schupp HT. Neuroimaging methods in affective neuroscience: selected methodological issues. Prog Brain Res 2006;156:31-51.

19. Junghöfer M, Schupp HT, Stark R, Vaitl D. Neuroimaging of emotion: empirical effects of proportional global signal scaling in fMRI data analysis. Neuroimage 2005;25(2):520-6.

20. Kissler J, Herbert C, Peyk P, Junghöfer M. Buzzwords: early cortical responses to emotional words during reading. Psychol Sci 2007;18(6):475-80.

21. Lang PJ, Bradley MM, Cuthbert BN. International affective picture system (IAPS): affective ratings of pictures and 
instruction manual. Technical Report A-6. University of Florida, Gainesville, FL; 2005.

22. Lang PJ, Bradley MM, Cuthbert BN. Emotion and motivation: measuring affective perception. J Clin Neurophysiol 1998;15(5):397-408.

23. Lang PJ, Bradley MM, Cuthbert BN. Emotion, motivation, and anxiety: brain mechanisms and psychophysiology. Biol Psychiatr 1998;44(12):1248-63.

24. Lewis S, Thoma RJ, Lanoue MD, Miller GA, Heller W, Edgar C, et al. Visual processing of facial affect. Neuroreport 2003;14(14):1841-5.

25. Liu L, Ioannides AA, Streit M. Single trial analysis of neurophysiological correlates of the recognition of complex objects and facial expressions of emotion. Brain Topogr 1999;11(4): 291-303.

26. Numminen J, Ahlfors S, Ilmoniemi R, Montonen J, Nenonen J. Transformation of multichannel magnetocardiographic signals to standard grid form. IEEE Trans Biomed Eng 1995;42(1): 72-8.

27. Öhman A. Face the beast and fear the face: animal and social fears as prototypes for evolutionary analyses of emotion. Psychophysiology 1986;23(2):123-45.

28. Öhman A, Flykt A, Esteves F. Emotion drives attention: detecting the snake in the grass. J Exp Psychol Gen 2001;130(3):466-78.
29. Peyk P, Schupp H, Keil A, Elbert TR, Junghöfer M. From ERPs to steady state: parallel processing of affective visual stimuli. Psychophysiology (under revision).

30. Putsche C, Dobel C, Schupp H, Kissler J, Pantev C, Junghöfer M. Odour conditioned faces attract preferential initial visual processing (submitted).

31. Rockstroh B, Junghöfer M, Elbert T, Buodo G, Miller GA. Electromagnetic brain activity evoked by affective stimuli in schizophrenia. Psychophysiology 2006;43(5):431-9.

32. Sabatinelli D, Bradley MM, Fitzsimmons JR, Lang PJ. Parallel amygdala and inferotemporal activation reflect emotional intensity and fear relevance. Neuroimage 2005;24(4):1265-70.

33. Schupp HT, Flaisch T, Stockburger J, Junghöfer M. Emotion and attention: event-related brain potential studies. Prog Brain Res 2006;156:31-51.

34. Schupp HT, Öhman A, Junghöfer M, Weike AI, Stockburger J, Hamm AO. The facilitated processing of threatening faces: an ERP analysis. Emotion 2004;4(2):189-200.

35. Streit M, Ioannides AA, Liu L, Wölwer W, Dammers J, Gross J, et al. Neurophysiological correlates of the recognition of facial expressions of emotion as revealed by magnetoencephalography. Brain research. Cognitive Brain Res 1999;7(4):481-91.

36. Vuilleumier P. How brains beware: neural mechanisms of emotional attention. Trends Cognitive Sci 2005;9:585-94. 